\title{
Jacobi spectral solution for weakly singular integral algebraic equations of index-1
}

Jingjun Zhao* and Shiying Wang

"Correspondence: hit_zjj@hit.edu.cn Department of Mathematics, Harbin Institute of Technology, Harbin, 150001, China

\begin{abstract}
This paper is concerned with obtaining the approximate solution of a class of semi-explicit Integral Algebraic equations (IAEs) of index-1 with weakly singular kernels. Some function transformations and variable transformations are used to change the equations into integral equations defined on the standard interval $[-1,1]$, so that the solution of the new system possesses better regularity and the Jacobi orthogonal polynomial theory can be applied conveniently. A Jacobi collocation method is proposed and its convergence analysis is provided, which theoretically justifies the spectral rate of convergence. The numerical results are given to verify our theoretical analysis.
\end{abstract}

MSC: 65 R20

Keywords: integral algebraic equations; Jacobi collocation method; system of weakly singular Volterra equations; index of IAEs; error analysis

\section{Introduction}

In this paper, we consider the following system of Volterra Integral equations (VIEs) with weakly singular kernels:

$$
\left\{\begin{array}{l}
y(t)=f_{1}(t)+\int_{0}^{t}(t-s)^{-\alpha} K_{11}(t, s) y(s) d s \\
\quad+\int_{0}^{t}(t-s)^{-\alpha} K_{12}(t, s) z(s) d s, \\
0=f_{2}(t)+\int_{0}^{t}(t-s)^{-\alpha} K_{21}(t, s) y(s) d s \quad t \in I=[0, T], \\
\quad+\int_{0}^{t}(t-s)^{-\alpha} K_{22}(t, s) z(s) d s,
\end{array}\right.
$$

where $0<\alpha=\frac{p}{q}<1(p, q \in \mathbb{N}, p<q)$, the functions $f_{1}, f_{2}$ and $K_{i j}(\cdot, \cdot)(i, j=1,2)$ are known to be smooth on $I$ and $D=\{(t, s): 0 \leq s \leq t \leq T\}$, respectively, and $y(t), z(t)$ are unknown solutions. We assume that $f_{2}(0)=0,\left|k_{22}(t, t)\right| \geq k_{0}>0, \forall t \in I$. Under these conditions, system (1.1) is called a system of Weakly Singular Integral Algebraic equations (WSIAEs) of index 1. System (1.1) can be written in the following form:

$$
A(t) X(t)=F(t)+\int_{0}^{t}(t-s)^{-\alpha} K(t, s) X(s) d s,
$$

where

$$
A=\left(\begin{array}{ll}
1 & 0 \\
0 & 0
\end{array}\right), \quad X(t)=(y(t), z(t))^{T}, \quad F(t)=\left(f_{1}(t), f_{2}(t)\right)^{T},
$$

○2014 Zhao and Wang; licensee Springer. This is an Open Access article distributed under the terms of the Creative Commons Attribution License (http://creativecommons.org/licenses/by/2.0), which permits unrestricted use, distribution, and reproduction in any medium, provided the original work is properly cited. 


$$
K(t, s)=\left(\begin{array}{ll}
K_{11}(t, s) & K_{12}(t, s) \\
K_{21}(t, s) & K_{22}(t, s)
\end{array}\right) .
$$

In practical applications, one frequently encounters system (1.1). A good source on applications of IAEs with weakly singular kernels is the initial (boundary) value problems for the semi-infinite strip and temperature boundary specification including two/three-phase inverse Stefan problems [1-4]. A wide ranging description of IAEs arising in applications is given in [5]. Several numerical methods for integral algebraic equations have been proposed (see e.g. [5-10]). However, as far as we know the numerical analysis of WSIAEs is largely incomplete and this is a new topic for research. The existence and uniqueness for the solution of WSIAEs has been given in $[11,12]$. The piecewise polynomial collocation method for system (1.1) and the concept of tractability index have been considered by Brunner [5]; he analyzed the regularity of the solutions using conditions that hold for the first and second kind Volterra integral equations. Recently, the effective numerical method based on the Chebyshev collocation scheme is designed for system (1.1) in [13] and its convergence analysis is provided.

The solutions of system (1.1) usually have a weak singularity at $t=0$, whose derivatives are unbounded at $t=0$. To overcome this difficulty, we use the idea of the authors in [14]. Both function transformation and variable transformation are used to change the system into a new system defined on the standard interval $[-1,1]$, so that the solutions of the new system possess better regularity and the Jacobi spectral collocation method can be applied conveniently. The aim of this work is to use the Jacobi collocation method to numerically solve the WSIAEs (1.1) and then a rigorous error analysis is provided in the weighted $L^{2}$-norm which shows the spectral rate of convergence is attained.

This paper is organized as follows. Some useful results for establishing the convergence results will be provided in Section 2. In Section 3, we carry out the Jacobi collocation approach for system (1.1). The convergence of the method in the weighted $L^{2}$-norm as a main result of the paper is given in Section 4. Section 5 gives some numerical experiments. The final section contains conclusions and remarks.

\section{Some useful results}

In this section, we discuss how the weakly singular IAEs can be changed to treat the problem. Furthermore, the index concept for WSIAEs which plays a fundamental role in both the analysis and the development of numerical algorithms for IAEs is discussed.

\subsection{Index definitions for WSIAEs}

There are several definitions of index in literature, not all completely equivalent, such as the tractability index (see, e.g. Definition (8.1.7) from [5]), the left index [6] and differentiation index [15]. Generally, the difficulties are arising in the theoretical and numerical analysis of IAEs relevant to the index notion.

In this paper, we use the concept of the differentiation index which measures how far the main WSIAE is apart from a regular system of VIEs. Namely, the number of analytical differentiations of system (1.1) until it can be formulated as a regular system of Volterra integral equations is called the differentiation index.

Now, we consider the index of system (1.1). According to the classical theory of Volterra integral equations with weakly singular kernels from [5, p.353], if we multiply both sides 
of the second equation of (1.1) by the factor $(u-t)^{\alpha-1}$ and integrate with respect to $t$, the following first kind Volterra integral equation with regular bounded kernels will be obtained:

$$
0=\int_{0}^{t} H_{21}(t, s) y(s) d s+\int_{0}^{t} H_{22}(t, s) z(s) d s+G_{\alpha}\left(f_{2}\right)
$$

where

$$
H_{21}(t, s)=\int_{0}^{1} \frac{K_{21}(s+(t-s) v, s)}{v^{\alpha}(1-v)^{1-\alpha}} d v, \quad H_{22}(t, s)=\int_{0}^{1} \frac{K_{22}(s+(t-s) v, s)}{v^{\alpha}(1-v)^{1-\alpha}} d v
$$

and

$$
G_{\alpha}\left(f_{2}\right)=\int_{0}^{t}(t-s)^{\alpha-1} f_{2}(s) d s .
$$

We get the following second kind integral equation by differentiation of equation (2.1):

$$
\begin{aligned}
0= & H_{21}(t, t) y(t)+H_{22}(t, t) z(t)+\int_{0}^{t} \frac{\partial H_{21}(t, s)}{\partial t} y(s) d s \\
& +\int_{0}^{t} \frac{\partial H_{22}(t, s)}{\partial t} z(s) d s+G_{\alpha}^{\prime}\left(f_{2}\right),
\end{aligned}
$$

where $H_{2 j}(t, t)=\frac{\sin (\alpha \pi)}{\pi} K_{2 j}(t, t)(j=1,2)$ and $G_{\alpha}^{\prime}\left(f_{2}\right)=\int_{0}^{t}(t-s)^{\alpha-1} f_{2}^{\prime}(s) d s$. In fact, $G_{\alpha}^{\prime}\left(f_{2}\right)$ can be obtained using integration by parts to $G_{\alpha}\left(f_{2}\right)$ with $f_{2}(0)=0$.

Because $\left|K_{22}(t, t)\right| \geq k_{0}>0$, we have $\left|H_{22}(t, t)\right|>0$, then equation (2.2) together with the first equation of (1.1) is a system of regular Volterra integral equation.

But it should be noted that this reduction (differentiation) is not useful from a numerical point of view and such a definition may be useful for understanding the underlying mathematical structure of a WSIAEs, and hence choosing a suitable numerical method for their solutions.

\subsection{Smoothness of the solution}

We assume that the Hölder space $C^{0, \beta}([0, T])=C^{\beta}([0, T])$ is defined to be a subspace of $C([0, T])$ that consists of functions which are Hölder continuous with the exponent $\beta$. For $m \in \mathbb{Z}_{+}$and $\beta \in(0,1]$, we similarly define the Hölder space

$$
C^{m, \beta}([0, T])=\left\{f \in C^{m}([0, T])\left|D^{v} f \in C^{0, \beta}([0, T]), \forall v,\right| v \mid=m\right\} .
$$

It is shown in [16] that this is a Banach space with the following norm:

$$
\|f\|_{C^{k, \beta}([0, T])}=\|f\|_{C^{m}([0, T])}+\sum_{|v|=m} \sup \left\{\frac{\left|D^{v} f(x)-D^{v} f(y)\right|}{|x-y|^{\beta}} \mid x, y \in[0, T], x \neq y\right\} .
$$

It is noted that the solutions of system (1.1) $y(t)$ and $z(t)$ lie in the Hölder space $C^{1-\alpha}(I)$ and $C^{\alpha}(I)$, respectively (see [5, Theorems 8.1.8, 6.1.6, and 6.1.14]). In other words, for any positive integer $m$, the solutions $y(t)$ and $z(t)$ do not belong to $C^{m}(I)$. It is well known that the spectral methods have been an efficient tool for solving the differential equations with 
smooth solutions. Using the idea of Li and Tang in [14], one can overcome this difficulty by introducing the following variable transformation:

$$
t=u^{q}, \quad u=\sqrt[q]{t}, \quad s=w^{q}, \quad w=\sqrt[q]{s},
$$

to change (1.1) to the following system:

$$
\left\{\begin{array}{c}
\hat{y}(u)=\hat{f}_{1}(u)+\int_{0}^{u}(u-w)^{-\alpha} \hat{K}_{11}(u, w) \hat{y}(w) d w \\
\quad+\int_{0}^{u}(u-w)^{-\alpha} \hat{K}_{12}(u, w) \hat{z}(w) d w \\
0=\hat{f}_{2}(u)+\int_{0}^{u}(u-w)^{-\alpha} \hat{K}_{21}(u, w) \hat{y}(w) d w \\
\quad+\int_{0}^{u}(u-w)^{-\alpha} \hat{K}_{22}(u, w) \hat{z}(w) d w
\end{array} \quad u \in[0, \sqrt[q]{T}],\right.
$$

where

$$
\begin{aligned}
& \hat{f}_{1}(u)=f_{1}\left(u^{q}\right), \quad \hat{f}_{2}(u)=f_{2}\left(u^{q}\right), \\
& \hat{K}_{i j}(u, w)=q w^{q-1}\left(u^{q-1}+u^{q-2} w+\cdots+w^{q-1}\right) K_{i j}\left(u^{q}, w^{q}\right) \quad(i, j=1,2),
\end{aligned}
$$

and $\hat{y}(u)=y\left(u^{q}\right), \hat{z}(u)=z\left(u^{q}\right)$ are the smooth solutions of system (2.4).

The existence and uniqueness results and the smoothness behavior of solutions $\hat{y}$ and $\hat{z}$ of system (2.4) can be obtained from the corresponding discussions of the classical theory of Volterra integral equations with weakly singular kernels from [5] (see e.g. Theorems 6.1.6 and 6.1.14 for further details).

\section{The Jacobi collocation method}

We first introduce some notations. Let $\omega^{\alpha, \beta}(x)=(1-x)^{\alpha}(1+x)^{\beta}(\alpha, \beta>-1)$ be a weight function in the usual sense. As defined in [17], let us denote $J_{n}^{\alpha, \beta}(x)$ the Jacobi polynomial of degree $n$ with respect to weight $\omega^{\alpha, \beta}(x)$. It is well known that the set of Jacobi polynomials $\left\{J_{n}^{\alpha, \beta}(x)\right\}_{n=0}^{\infty}$ forms a complete $L_{\omega^{\alpha, \beta}}^{2}(-1,1)$ orthogonal system, where $L_{\omega^{\alpha, \beta}}^{2}(-1,1)$ is the space

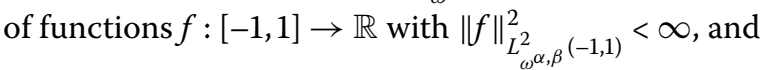

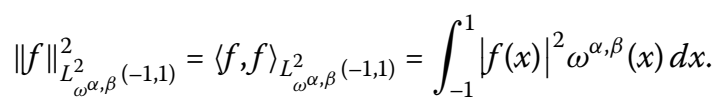

Particularly, when $\alpha=\beta=0$, the Jacobi polynomials become Legendre polynomials, and when $\alpha=\beta=-\frac{1}{2}$, the Jacobi polynomials become Chebyshev polynomials.

For the sake of obtaining a Jacobi spectral method for system (2.4), we employ the linear transformation

$$
x=\frac{2}{\sqrt[q]{T}} w-1, \quad-1 \leq x \leq v, \quad v=\frac{2}{\sqrt[q]{T}} u-1, \quad-1 \leq v \leq 1,
$$

to rewrite system (2.4) as follows:

$$
\left\{\begin{array}{c}
\tilde{y}(v)=\tilde{f}_{1}(u)+\int_{-1}^{v}(v-x)^{-\alpha} \tilde{K}_{11}(v, x) \tilde{y}(x) d x \\
\quad+\int_{-1}^{v}(v-x)^{-\alpha} \tilde{K}_{12}(v, x) \tilde{z}(x) d x, \quad v \in[-1,1] \\
0=\tilde{f}_{2}(u)+\int_{-1}^{v}(v-x)^{-\alpha} \tilde{K}_{21}(v, x) \tilde{y}(x) d x \quad \\
\quad+\int_{-1}^{v}(v-x)^{-\alpha} \tilde{K}_{22}(v, x) \tilde{z}(x) d x
\end{array}\right.
$$


where $\tilde{f}_{1}(v)=\hat{f}_{1}\left(\frac{q}{2}(v+1)\right), \tilde{f}_{2}(v)=\hat{f}_{2}\left(\frac{q}{2}(v+1)\right), \tilde{y}(v)=\hat{y}\left(\frac{q}{2}(v+1)\right), \tilde{z}(v)=\hat{z}\left(\frac{q}{2}(v+1)\right)$, and

$$
\tilde{K}_{i j}(v, x)=\left(\frac{\sqrt[q]{T}}{2}\right)^{1-\alpha} \hat{K}_{i j}\left(\frac{\sqrt[q]{T}}{2}(v+1), \frac{q}{2}(x+1)\right) \quad(i, j=1,2)
$$

It is well known that, in the Jacobi collocation method, we use $\tilde{y}_{N}$ and $\tilde{z}_{N}$ of the following form:

$$
\begin{aligned}
& \tilde{y}_{N}=I_{N}(\tilde{y}(v))=\sum_{k=0}^{N} \tilde{y}\left(v_{k}\right) L_{k}(v), \\
& \tilde{z}_{N}=I_{N}(\tilde{z}(v))=\sum_{k=0}^{N} \tilde{z}\left(v_{k}\right) L_{k}(v),
\end{aligned}
$$

to approximate the solutions $\tilde{y}$ and $\tilde{z}$, where $v_{k}$ are the Gauss-Jacobi quadrature points and $L_{k}$ are the interpolating Lagrange polynomials

$$
L_{k}(v)=\frac{J_{N+1}^{\alpha, \beta}(v)}{\left(v-v_{k}\right) J_{N+1}^{\alpha, \beta}(v)}, \quad k=0,1, \ldots, N
$$

where $J_{N+1}^{\alpha, \beta}(v)$ is the $(N+1)$ th-order Jacobi polynomial.

Now, we fix the value of $v$ for general kernels $\tilde{K}_{i j}(v, x)$ and choose $x=v_{k}$, then $\tilde{K}_{i j}(v, x)$ can be approximated by a univariate Lagrange interpolating polynomial as follows:

$$
I_{N}\left(\tilde{K}_{i j}(v, x)\right)=\sum_{k=0}^{N} \tilde{K}_{i j}\left(v, v_{k}\right) L_{k}(x), \quad i, j=1,2 .
$$

Substituting equations (3.3) and (3.5) into (3.2) and inserting the collocation points $v_{k}$ $(k=0,1, \ldots, N)$ in the obtained equation, one obtains the following system of linear equations:

$$
\begin{aligned}
& \tilde{y}\left(v_{k}\right)= \tilde{f}_{1}\left(v_{k}\right)+\int_{-1}^{v_{k}}\left(v_{k}-x\right)^{-\alpha} I_{N}\left(\tilde{K}_{11}\left(v_{k}, x\right)\right) \tilde{y}(x) d x \\
&+\int_{-1}^{v_{k}}\left(v_{k}-x\right)^{-\alpha} I_{N}\left(\tilde{K}_{12}\left(v_{k}, x\right)\right) \tilde{z}(x) d x \\
& 0=\tilde{f}_{2}\left(v_{k}\right)+\int_{-1}^{v_{k}}(v-x)^{-\alpha} I_{N}\left(\tilde{K}_{21}\left(v_{k}, x\right)\right) \tilde{y}(x) d x \\
&+\int_{-1}^{v_{k}}\left(v_{k}-x\right)^{-\alpha} I_{N}\left(\tilde{K}_{22}\left(v_{k}, x\right)\right) \tilde{z}(x) d x \quad(k=0,1, \ldots, N) .
\end{aligned}
$$

So, the unknown coefficients $\tilde{y}\left(v_{0}\right), \tilde{y}\left(v_{1}\right), \ldots, \tilde{y}\left(v_{N}\right)$ and $\tilde{z}\left(v_{0}\right), \tilde{z}\left(v_{1}\right), \ldots, \tilde{z}\left(v_{N}\right)$ are obtained by solving the linear system (3.6) and finally the approximate solutions $\tilde{y}_{N}$ and $\tilde{z}_{N}$ will be computed by substituting these coefficients into (3.3).

\section{Error estimate}

To prove the error estimate in the weighted $L^{2}$-norm, we first introduce some lemmas which are usually required to obtain the convergence results. 
Lemma 1 ([18]) Let $I_{N}$ be a linear operator from $C^{k, \beta}([-1,1])$ to $P_{N}$, then $\forall k \in \mathbb{N}, \beta \in$ $[0,1]$, there exists a positive constant $C_{k, \beta}$, such that $\forall f \in C^{k, \beta}([-1,1]), \exists I_{N} f \in P_{N}$, s.t., $\| f-$ $I_{N} f\left\|_{L^{\infty}} \leq C_{k, \beta} N^{-(k+\beta)}\right\| f \|_{C^{k, \beta}([-1,1])}$.

Lemma $2([14])$ Let $\left\{L_{j}(x)\right\}_{j=0}^{N}$ be Lagrange interpolation polynomials with the Jacobi Gauss points $\left\{x_{j}\right\}_{j=0}^{N}$, then

$$
\left\|I_{N}\right\|_{L^{\infty}}=\max _{x \in(-1,1)} \sum_{i=0}^{N}\left|L_{i}(x)\right|=\left\{\begin{array}{ll}
O(\log N), & -1<\alpha, \beta \leq-\frac{1}{2}, \\
O\left(N^{\frac{1}{2}+\gamma}\right), & \text { otherwise, }
\end{array} \quad \gamma=\max \{\alpha, \beta\} .\right.
$$

Let $e_{1}(v)=\tilde{y}_{N}(v)-\tilde{y}(v), e_{2}(v)=\tilde{z}_{N}(v)-\tilde{z}(v)$ denote the error functions. The following main theorem reveals the convergence results of the presented scheme in the weighted $L^{2}$-norm.

Theorem 1 Consider the index-1 weakly singular integral algebraic equations (1.1) and its transformed representation (3.2). Let $\tilde{D}=\{(t, s):-1 \leq x \leq v \leq T\},\left(\tilde{y}_{N}, \tilde{z}_{N}\right)$ be the approximated solution for the exact solution $(\tilde{y}, \tilde{z})$ of $(3.2)$ based on the spectral collocation scheme (3.6) and suppose the following conditions hold:

(1) $\tilde{K}_{1 j} \in C^{m}(\tilde{D})(j=1,2), \tilde{f}_{1} \in C^{m}[-1,1]$;

(2) $\tilde{K}_{2 j} \in C^{m+1}(\tilde{D})(j=1,2), \tilde{f}_{2} \in C^{m+1}[-1,1]$ with $\tilde{f}_{2}(-1)=0$;

(3) $\left|\tilde{K}_{22}(v, v)\right|>0, \forall v \in[-1,1]$.

Then for sufficiently large $N$,

$$
\begin{aligned}
& \left\|e_{1}\right\|_{L^{\alpha, \beta}}^{2}(-1,1)=\left\|\tilde{y}_{N}-\tilde{y}\right\|_{L^{\alpha}{ }^{\alpha, \beta}(-1,1)} \leq \begin{cases}O\left(N^{\frac{1}{2}-m} \log N\right), & -1<\alpha, \beta \leq-\frac{1}{2}, \\
O\left(N^{1+\gamma-m} \log N\right), & \text { otherwise, }\end{cases} \\
& \left\|e_{2}\right\|_{L^{\alpha, \beta}(-1,1)}^{2}=\left\|\tilde{z}_{N}-\tilde{z}\right\|_{L^{2}{ }^{\alpha, \beta}(-1,1)} \leq \begin{cases}O\left(N^{\frac{5}{2}-m} \log N\right), & -1<\alpha, \beta \leq-\frac{1}{2}, \\
O\left(N^{3+\gamma-m} \log N\right), & \text { otherwise, }\end{cases}
\end{aligned}
$$

where $\gamma=\max \{\alpha, \beta\}$.

Proof First, the first equation of system (3.6) can be rewritten as

$$
\tilde{y}\left(v_{k}\right)=\tilde{f}_{1}\left(v_{k}\right)+\sum_{n=0}^{N} \sum_{l=0}^{N}\left(a_{n l}+b_{n l}\right) W_{n l}\left(v_{k}\right)
$$

where

$$
a_{n l}=\left\{\begin{array}{ll}
\tilde{y}_{n}\left(\tilde{K}_{11}\right)_{n}, & n=l, \\
\tilde{y}_{n}\left(\tilde{K}_{11}\right)_{l}+\tilde{y}_{l}\left(\tilde{K}_{11}\right)_{n}, & n \neq l,
\end{array} \quad b_{n l}= \begin{cases}\tilde{z}_{n}\left(\tilde{K}_{12}\right)_{n}, & n=l, \\
\tilde{z}_{n}\left(\tilde{K}_{12}\right)_{l}+\tilde{z}_{l}\left(\tilde{K}_{12}\right)_{n}, & n \neq l,\end{cases}\right.
$$

and

$$
\begin{aligned}
& \left(\tilde{K}_{i j}\right)_{n}=\tilde{K}_{i j}\left(v_{k}, v_{n}\right) \quad(i, j=1,2), \quad \tilde{y}_{n}=\tilde{y}\left(v_{n}\right), \\
& W_{n l}\left(v_{k}\right)=\int_{-1}^{v_{k}}\left(v_{k}-x\right)^{-\alpha} L_{n}(x) L_{l}(x) d x .
\end{aligned}
$$


Using these notations, equation (4.1) can be written as

$$
\begin{aligned}
\tilde{y}\left(v_{k}\right)= & \tilde{f}_{1}\left(v_{k}\right)+\int_{-1}^{v_{k}}\left(v_{k}-x\right)^{-\alpha}\left(\tilde{K}_{11}\left(v_{k}, x\right) e_{1}(x)+\tilde{K}_{12}\left(v_{k}, x\right) e_{2}(x)\right) d x \\
& +\int_{-1}^{v_{k}}\left(v_{k}-x\right)^{-\alpha}\left(\tilde{K}_{11}\left(v_{k}, x\right) \tilde{y}(x)+\tilde{K}_{12}\left(v_{k}, x\right) \tilde{z}(x)\right) d x+Z_{1}\left(v_{k}\right)+Z_{2}\left(v_{k}\right),
\end{aligned}
$$

where

$$
\begin{aligned}
& Z_{1}\left(v_{k}\right)=\sum_{n=0}^{N} \sum_{l=0}^{N} a_{n l} W_{n l}\left(v_{k}\right)-\int_{-1}^{v_{k}}\left(v_{k}-x\right)^{-\alpha} \tilde{K}_{11}\left(v_{k}, x\right) I_{N}(\tilde{y}(x)) d x \\
& Z_{2}\left(v_{k}\right)=\sum_{n=0}^{N} \sum_{l=0}^{N} b_{n l} W_{n l}\left(v_{k}\right)-\int_{-1}^{v_{k}}\left(v_{k}-x\right)^{-\alpha} \tilde{K}_{12}\left(v_{k}, x\right) I_{N}(\tilde{z}(x)) d x .
\end{aligned}
$$

Multiplying equation (4.2) by $L_{k}(v)$ and summing up from 0 to $N$, one obtains

$$
\begin{aligned}
\tilde{y}_{N}(v)= & I_{N}\left(\tilde{f}_{1}(v)\right)+I_{N}\left(\int_{-1}^{v}\left(v_{k}-x\right)^{-\alpha}\left(\tilde{K}_{11}(v, x) e_{1}(x)+\tilde{K}_{12}(v, x) e_{2}(x)\right) d x\right) \\
& +I_{N}\left(\int_{-1}^{v}(v-x)^{-\alpha}\left(\tilde{K}_{11}(v, x) \tilde{y}(x)+\tilde{K}_{12}(v, x) \tilde{z}(x)\right) d x\right) \\
& +I_{N}\left(Z_{1}(v)\right)+I_{N}\left(Z_{2}(v)\right),
\end{aligned}
$$

and subtracting equation (4.4) from the first equation of (3.2), we have

$$
\begin{aligned}
e_{1}(v)= & I_{N}\left(\int_{-1}^{v}(v-x)^{-\alpha}\left(\tilde{K}_{11}(v, x) e_{1}(x)+\tilde{K}_{12}(v, x) e_{2}(x)\right) d x\right) \\
& +W_{1}+W_{2}+W_{3}+I_{N}\left(Z_{1}(v)\right)+I_{N}\left(Z_{2}(v)\right)
\end{aligned}
$$

where

$$
\begin{aligned}
& W_{1}=I_{N}\left(\int_{-1}^{v}(v-x)^{-\alpha} \tilde{K}_{11}(v, x) \tilde{y}(x) d x\right)-\int_{-1}^{v}(v-x)^{-\alpha} \tilde{K}_{11}(v, x) \tilde{y}(x) d x, \\
& W_{2}=I_{N}\left(\int_{-1}^{v}(v-x)^{-\alpha} \tilde{K}_{12}(v, x) \tilde{z}(x) d x\right)-\int_{-1}^{v}(v-x)^{-\alpha} \tilde{K}_{12}(v, x) \tilde{z}(x) d x, \\
& W_{3}=I_{N}\left(\tilde{f}_{1}(v)\right)-\tilde{f}_{1}(v) .
\end{aligned}
$$

Equation (4.5) can be rewritten as follows:

$$
\begin{aligned}
e_{1}(v)= & \int_{-1}^{v}(v-x)^{-\alpha}\left(\tilde{K}_{11}(v, x) e_{1}(x)+\tilde{K}_{12}(v, x) e_{2}(x)\right) d x \\
& +W_{1}+W_{2}+W_{3}+W_{4}+W_{5}+I_{N}\left(Z_{1}(v)\right)+I_{N}\left(Z_{2}(v)\right),
\end{aligned}
$$

where

$$
W_{4}=I_{N}\left(\int_{-1}^{v}(v-x)^{-\alpha} \tilde{K}_{11}(v, x) e_{1}(x) d x\right)-\int_{-1}^{v}(v-x)^{-\alpha} \tilde{K}_{11}(v, x) e_{1}(x) d x
$$




$$
W_{5}=I_{N}\left(\int_{-1}^{v}(v-x)^{-\alpha} \tilde{K}_{12}(v, x) e_{2}(x) d x\right)-\int_{-1}^{v}(v-x)^{-\alpha} \tilde{K}_{12}(v, x) e_{2}(x) d x .
$$

Next, by using the second equation of (3.2), we have

$$
I_{N}\left(\int_{-1}^{v}(v-x)^{-\alpha}\left(\tilde{K}_{21}(v, x) \tilde{y}(x)+\tilde{K}_{22}(v, x) \tilde{z}(x)\right) d x\right)=-I_{N}(\tilde{g}(v)) .
$$

For the second equation of (3.6), using a similar procedure as outlined for obtaining the relation (4.4), and then inserting equation (4.7) into the resulting equation, we get

$$
\begin{aligned}
0= & \int_{-1}^{v}(v-x)^{-\alpha}\left(\tilde{K}_{21}(v, x) e_{1}(x)+\tilde{K}_{22}(v, x) e_{2}(x)\right) d x \\
& +W_{6}+W_{7}+I_{N}\left(Z_{3}(v)\right)+I_{N}\left(Z_{4}(v)\right),
\end{aligned}
$$

where

$$
\begin{aligned}
& W_{6}=I_{N}\left(\int_{-1}^{v}(v-x)^{-\alpha} \tilde{K}_{21}(v, x) e_{1}(x) d x\right)-\int_{-1}^{v}(v-x)^{-\alpha} \tilde{K}_{21}(v, x) e_{1}(x) d x, \\
& W_{7}=I_{N}\left(\int_{-1}^{v}(v-x)^{-\alpha} \tilde{K}_{22}(v, x) e_{2}(x) d x\right)-\int_{-1}^{v}(v-x)^{-\alpha} \tilde{K}_{22}(v, x) e_{2}(x) d x, \\
& Z_{3}\left(v_{k}\right)=\sum_{n=0}^{N} \sum_{l=0}^{N} c_{n l} W_{n l}\left(v_{k}\right)-\int_{-1}^{v_{k}}\left(v_{k}-x\right)^{-\alpha} \tilde{K}_{21}\left(v_{k}, x\right) I_{N}(\tilde{y}(x)) d x, \\
& Z_{4}\left(v_{k}\right)=\sum_{n=0}^{N} \sum_{l=0}^{N} d_{n l} W_{n l}\left(v_{k}\right)-\int_{-1}^{v_{k}}\left(v_{k}-x\right)^{-\alpha} \tilde{K}_{22}\left(v_{k}, x\right) I_{N}(\tilde{z}(x)) d x,
\end{aligned}
$$

and

$$
c_{n l}=\left\{\begin{array}{ll}
\tilde{y}_{n}\left(\tilde{K}_{21}\right)_{n}, & n=l, \\
\tilde{y}_{n}\left(\tilde{K}_{21}\right)_{l}+\tilde{y}_{l}\left(\tilde{K}_{21}\right)_{n}, & n \neq l,
\end{array} \quad d_{n l}= \begin{cases}\tilde{z}_{n}\left(\tilde{K}_{22}\right)_{n}, & n=l \\
\tilde{z}_{n}\left(\tilde{K}_{22}\right)_{l}+\tilde{z}_{l}\left(\tilde{K}_{22}\right)_{n}, & n \neq l .\end{cases}\right.
$$

Using a similar method for obtaining equation (2.1) in Section 2, equation (4.8) can be written as

$$
0=\int_{-1}^{v} \tilde{H}_{21}(v, x) e_{1}(x) d x+\int_{-1}^{v} \tilde{H}_{22}(v, x) e_{2}(x) d x+W_{6}+W_{7}+G_{\alpha}(F),
$$

where $F=W_{6}+W_{7}+I_{N}\left(Z_{3}(v)\right)+I_{N}\left(Z_{4}(v)\right), G_{\alpha}(F)=\int_{-1}^{v}(v-x)^{\alpha-1} F(x) d x$.

On differentiation of equation (4.9) with respect to $v$, we obtain a second kind integral equation as follows:

$$
\begin{aligned}
& -\tilde{H}_{21}(v, v) e_{1}(v)-\tilde{H}_{22}(v, v) e_{2}(v) \\
& \quad=\int_{-1}^{v} \frac{\partial \tilde{H}_{21}(v, v)}{\partial v} e_{1}(x) d x+\int_{-1}^{v} \frac{\partial \tilde{H}_{22}(v, v)}{\partial v} e_{2}(x) d x+G_{\alpha}^{\prime}(F),
\end{aligned}
$$

where

$$
G_{\alpha}^{\prime}(F)=\int_{-1}^{v}(v-x)^{\alpha-1} F^{\prime}(x) d x .
$$


Now, we rewrite equation (4.10) as

$$
\begin{aligned}
& -\tilde{H}_{21}(v, v) e_{1}(v)-\tilde{H}_{22}(v, v) e_{2}(v) \\
& =\int_{-1}^{v}(v-x)^{-\alpha}\left((v-x)^{\alpha} \frac{\partial \tilde{H}_{21}(v, v)}{\partial v} e_{1}(x)\right) d x \\
& \quad+\int_{-1}^{v}(v-x)^{-\alpha}\left((v-x)^{\alpha} \frac{\partial \tilde{H}_{22}(v, v)}{\partial v} e_{2}(x)\right) d x+G_{\alpha}^{\prime}(F),
\end{aligned}
$$

equations (4.6) and (4.12) can be written in the form of the compact matrix representation:

$$
H(v, v) E(v)=\int_{-1}^{v}(v-x)^{-\alpha} R(v, x) E(x) d x+M
$$

where

$$
\begin{gathered}
H(v, v)=\left[\begin{array}{cc}
1 & 0 \\
-\tilde{H}_{21}(v, v) & -\tilde{H}_{22}(v, v)
\end{array}\right], \quad R(v, x)=\left[\begin{array}{cc}
\tilde{K}_{11}(v, x) & \tilde{K}_{12}(v, x) \\
(v-x)^{\alpha} \frac{\partial \tilde{K}_{21}(v, x)}{\partial v} & (v-x)^{\alpha} \frac{\partial \tilde{K}_{22}(v, x)}{\partial v}
\end{array}\right], \\
E(v)=\left(\begin{array}{c}
e_{1}(v) \\
e_{2}(v)
\end{array}\right), \quad M=\left(\begin{array}{c}
W_{1}+W_{2}+W_{3}+W_{4}+W_{5}+I_{N}\left(Z_{1}(v)\right)+I_{N}\left(Z_{2}(v)\right) \\
G_{\alpha}^{\prime}(F)
\end{array}\right) .
\end{gathered}
$$

From equation (2.2), $\tilde{H}_{21}(v, v)=\frac{\sin \alpha \pi}{\pi} \tilde{K}_{21}(v, v)$ and $\tilde{H}_{22}(v, v)=\frac{\sin \alpha \pi}{\pi} \tilde{K}_{22}(v, v)$, and from the condition (3) of Theorem $1,\left|\tilde{K}_{22}(v, v)\right|>0, \forall v \in[-1,1]$, so the matrix $H(v, v)$ is invertible and its inverse can be written as

$$
H^{-1}(v, v)=\left[\begin{array}{cc}
1 & 0 \\
-\tilde{K}_{21}(v, v) & \frac{-\pi}{\sin (\alpha \pi) \tilde{K}_{22}(v, v)}
\end{array}\right] .
$$

Multiplying equation (4.13) by $H^{-1}(v, v)$ yields

$$
\|E(v)\|_{L_{\omega}^{2}(-1,1)} \leq \Phi \int_{-1}^{v}(v-x)^{-\alpha}\|E(v)\|_{L_{\omega}^{2}(-1,1)} d x+\|\hat{N}\|_{L_{\omega}^{2}(-1,1)}
$$

where $\Phi=\max _{-1 \leq x<v \leq 1}\left\|H^{-1}(v, v) R(v, x)\right\|_{L_{\omega}^{2}(-1,1)}$ and $\hat{N}=H^{-1}(v, v) M$.

By the generalized Gronwall inequality [19, Lemma (3.6)], one obtains

$$
\|E(v)\|_{L_{\omega}^{2}(-1,1)} \leq \Phi \int_{-1}^{v}(v-x)^{-\alpha}\|\hat{N}\|_{L_{\omega}^{2}(-1,1)} d x+\|\hat{N}\|_{L_{\omega}^{2}(-1,1)} .
$$

It follows from the generalized Hardy inequality [14, Lemma 5] that

$$
\|E(v)\|_{L_{\omega}^{2}(-1,1)} \leq C\|\hat{N}\|_{L_{\omega}^{2}(-1,1)}
$$

From now on, for simplicity, we denote $\|\cdot\|_{L_{\omega}^{2}(-1,1)}$ by $\|\cdot\|$ and try to derive the error bounds for the proposed method step by step.

Step 1: We now estimate the error bounds for $W_{i}(i=1, \ldots, 5)$. Since $I_{N}$ denotes the interpolation operator, we have

$$
I_{N} \varphi(x)=\varphi(x), \quad \forall \varphi(x) \in \mathrm{P}_{N}
$$


It is noted that

$$
\begin{aligned}
\left\|W_{4}\right\| & =\left\|I_{N} \Gamma e_{1}-\Gamma e_{1}\right\|=\left\|I_{N} \Gamma e_{1}-I_{N}\left(I_{N}\left(\Gamma e_{1}\right)\right)+I_{N}\left(\Gamma e_{1}\right)-\Gamma e_{1}\right\| \\
& \leq\left\|I_{N}\right\|\left\|\Gamma e_{1}-I_{N}\left(\Gamma e_{1}\right)\right\|+\left\|I_{N}\left(\Gamma e_{1}\right)-\Gamma e_{1}\right\| \\
& \leq\left(\left\|I_{N}\right\|_{L^{\infty}}+1\right)\left\|\Gamma e_{1}-I_{N}\left(\Gamma e_{1}\right)\right\|_{L^{\infty}},
\end{aligned}
$$

where $I_{N}$ is defined in Lemma 1 , and

$$
\Gamma e_{1}=\int_{-1}^{v}(v-x)^{-\alpha} \tilde{K}_{11}(v, x) e_{1}(x) d x
$$

Using Lemma 1 for $k=0$, Lemma 2, and Lemma (3.5) from [19], we obtain

$$
\left\|W_{4}\right\| \leq \begin{cases}C(\log N+1) N^{-\beta}\left\|\Gamma e_{1}\right\|_{C^{0, \beta([-1,1])}} \leq C \log N N^{-\beta}\left\|e_{1}\right\|_{L^{\infty}}, & -1<\alpha, \beta \leq \frac{1}{2} \\ C\left(N^{\frac{1}{2}+\gamma}+1\right) N^{-\beta}\left\|\Gamma e_{1}\right\|_{C^{0, \beta([-1,1])}} \leq C N^{\frac{1}{2}+\gamma} N^{-\beta}\left\|e_{1}\right\|_{L^{\infty}}, & \text { otherwise. }\end{cases}
$$

Then using the inequality (5.5.28) from [17], we have

$$
\left\|W_{4}\right\| \leq \begin{cases}C \log N N^{\frac{1}{2}-m-\beta}|\tilde{y}|_{H_{\omega}^{m, N}(-1,1)}, & -1<\alpha, \beta \leq \frac{1}{2} \\ C N^{1+\gamma-\beta-m}|\tilde{y}|_{H_{\omega}^{m, N}(-1,1)}, & \text { otherwise }\end{cases}
$$

Here

$$
|\tilde{y}|_{H_{\omega}^{m, N}(-1,1)}=\left(\sum_{j=\min (m, N+1)}^{m}\left\|\tilde{y}^{(j)}\right\|_{L_{\omega}^{2}(-1,1)}\right)^{\frac{1}{2}} .
$$

Similarly,

$$
\begin{aligned}
&\left\|W_{5}\right\| \leq \begin{cases}C \log N N^{\frac{1}{2}-m-\beta}|\tilde{z}|_{H_{\omega}^{m, N}(-1,1)}, & -1<\alpha, \beta \leq 1 / 2, \\
C N^{1+\gamma-\beta-m}|\tilde{z}|_{H_{\omega}^{m, N}(-1,1)} & \text { otherwise, }\end{cases} \\
&\left\|W_{1}\right\| \leq \begin{cases}C \log N N^{-\beta}\|\tilde{y}\|_{L^{\infty}}, & -1<\alpha, \beta \leq 1 / 2, \\
C N^{\frac{1}{2}+\gamma-\beta}\|\tilde{y}\|_{L^{\infty}}, & \text { otherwise, }\end{cases} \\
&\left\|W_{2}\right\| \leq \begin{cases}C \log N N^{-\beta}\|\tilde{z}\|_{L^{\infty}}, & -1<\alpha, \beta \leq 1 / 2, \\
C N^{\frac{1}{2}+\gamma-\beta}\|\tilde{z}\|_{L^{\infty}}, & \text { otherwise, }\end{cases} \\
&\left\|W_{3}\right\| \leq C N^{-m}|\tilde{f}|_{H_{\omega}^{m, N}(-1,1)} .
\end{aligned}
$$

Step 2: In this step, we estimate $I_{N}\left(Z_{1}(v)\right)$ and $I_{N}\left(Z_{2}(v)\right)$. Using Lemma 2, we have

$$
\begin{aligned}
\left\|I_{N}\left(Z_{1}(v)\right)\right\| & \leq \max _{0 \leq k \leq N}\left|Z_{1}\left(v_{k}\right)\right|\left\|I_{N}\right\|_{L^{\infty}} \\
& \leq \max _{0 \leq k \leq N}\left|Z_{1}\left(v_{k}\right)\right| \cdot \begin{cases}O(\log N), & -1<\alpha, \beta \leq-\frac{1}{2}, \\
O\left(N^{\frac{1}{2}+\gamma}\right), & \text { otherwise. }\end{cases}
\end{aligned}
$$


Furthermore, from (4.3) we obtain

$$
\begin{aligned}
\left|Z_{1}\left(v_{k}\right)\right| & =\left|\int_{-1}^{v_{k}}\left(v_{k}-x\right)^{-\alpha}\left(I_{N}\left(\tilde{K}_{11}\left(v_{k}, x\right)\right)-\tilde{K}_{11}\left(v_{k}, x\right) \tilde{y}_{N}(x)\right) d x\right| \\
& \leq C\left\|I_{N}\left(\tilde{K}_{11}\left(v_{k}, x\right)\right)-\tilde{K}_{11}\left(v_{k}, x\right)\right\|_{L^{\infty}}\left\|\tilde{y}_{N}\right\|_{L^{\infty}} \int_{-1}^{v_{k}}\left(v_{k}-x\right)^{-\alpha} d x .
\end{aligned}
$$

Using the transformation (3.1), we have

$$
\int_{-1}^{v_{k}}\left(v_{k}-x\right)^{-\alpha} d x=\left(v_{k}+1\right)^{(1-\alpha)} B(1,1-\alpha)
$$

where $B(\cdot, \cdot)$ denotes the Euler Beta function. Then using the inequality (5.5.28) from [17], we have

$$
\left|Z_{1}\left(v_{k}\right)\right| \leq C\left(v_{k}+1\right)^{(1-\alpha)} B(1,1-\alpha) N^{\frac{1}{2}-m}\left|\tilde{K}_{11}\left(v_{k}, x\right)\right|_{H_{\omega}^{m, N}(-1,1)}\left\|\tilde{y}_{N}\right\|_{L^{\infty}} .
$$

From (4.21), we obtain

$$
\left\|I_{N}\left(Z_{1}(v)\right)\right\| \leq\left\{\begin{array}{l}
C \log N N^{\frac{1}{2}-m} \Phi_{11}\left\|\tilde{y}_{N}\right\|_{L^{\infty}}, \\
C N^{1+\gamma-m} \Phi_{11}\left\|\tilde{y}_{N}\right\|_{L^{\infty}},
\end{array}\right.
$$

where

$$
\Phi_{i j}=B(1,1-\alpha) \max _{0 \leq k \leq N}\left(v_{k}+1\right)^{(1-\alpha)}\left|\tilde{K}_{i j}\left(v_{k}, x\right)\right|_{H_{\omega}^{m, N}(-1,1)} \quad(i, j=1,2) .
$$

Similarly, we have

$$
\left\|I_{N}\left(Z_{2}(v)\right)\right\| \leq\left\{\begin{array}{l}
C \log N N^{\frac{1}{2}-m} \Phi_{12}\left\|\tilde{y}_{N}\right\|_{L^{\infty}}, \\
C N^{1+\gamma-m} \Phi_{12}\left\|\tilde{y}_{N}\right\|_{L^{\infty}} .
\end{array}\right.
$$

Step 3: Here, our aim is to find a bound for $G_{\alpha}^{\prime}(F)$ using the suitable inequalities as well as the previously obtained bounds. Therefore, we estimate equation (4.11) as

$$
\left|G_{\alpha}^{\prime}(F)\right| \leq \int_{-1}^{v}(v-x)^{\alpha-1}\left|F^{\prime}(x)\right| d x .
$$

By using the generalized Hardy inequality from [14], it can be seen that

$$
\left\|G_{\alpha}^{\prime}(F)\right\| \leq C\left\|F^{\prime}(x)\right\| \leq C\left(\left\|W_{6}^{\prime}\right\|+\left\|W_{7}^{\prime}\right\|+\left\|I_{N}\left(Z_{3}^{\prime}(v)\right)\right\|+\left\|I_{N}\left(Z_{4}^{\prime}(v)\right)\right\|\right) .
$$

Using the inequality (5.5.25) from [17], we have

$$
\left\|W_{6}^{\prime}\right\| \leq C N^{1-m}\left|\tilde{\Gamma} e_{1}\right|_{H_{\omega}^{m, N}(-1,1)},
$$

where $\Gamma e_{1}=\int_{-1}^{v}(v-x)^{-\alpha} \tilde{K}_{21}(v, x) e_{1}(x) d x$.

Let $m=1$ in (4.19), we have

$$
\left\|W_{6}^{\prime}\right\| \leq C\left\|\frac{\partial \Gamma e_{1}}{\partial v}\right\|
$$


Moreover, using integration by parts and the generalized Hardy inequality, we have

$$
\left\|\frac{\partial \Gamma e_{1}}{\partial v}\right\| \leq C\left(\left\|e_{1}\right\|+\left\|e_{1}^{\prime}\right\|\right)
$$

Now, using the inequalities (5.5.22) and (5.5.25) from [17] yields

$$
\left\|W_{6}^{\prime}\right\| \leq C\left(N^{-m}+N^{1-m}\right)|\tilde{y}|_{H_{\omega}^{m, N}(-1,1)} .
$$

Similarly,

$$
\left\|W_{7}^{\prime}\right\| \leq C\left(N^{-m}+N^{1-m}\right)|\tilde{z}|_{H_{\omega}^{m, N}(-1,1)} .
$$

On the other hand, using the inequality (5.5.4) from [17] and equation (4.22), we obtain

$$
\left\|I_{N}^{\prime}\left(Z_{3}(v)\right)\right\| \leq C N^{2}\left\|I_{N}\left(Z_{3}(v)\right)\right\| \leq\left\{\begin{array}{l}
C \log N N^{\frac{5}{2}-m} \Phi_{21}\left\|\tilde{y}_{N}\right\|_{L^{\infty}}, \\
C N^{3+\gamma-m} \Phi_{21}\left\|\tilde{y}_{N}\right\|_{L^{\infty}} .
\end{array}\right.
$$

Also we have

$$
\left\|I_{N}^{\prime}\left(Z_{4}(v)\right)\right\| \leq C N^{2}\left\|I_{N}\left(Z_{4}(v)\right)\right\| \leq\left\{\begin{array}{l}
C \log N N^{\frac{5}{2}-m} \Phi_{22}\left\|\tilde{y}_{N}\right\|_{L^{\infty}}, \\
C N^{3+\gamma-m} \Phi_{22}\left\|\tilde{y}_{N}\right\|_{L^{\infty}} .
\end{array}\right.
$$

Finally, the above estimates together with equation (4.16) lead to Theorem 1.

It is noted in [20] that the function transformation generally makes the resulting equations and approximations more complicated. As discussed in [14, p.80], we can also obtain the error estimates for the numerical solutions to the WSIAEs (1.1).

Theorem 2 Consider the index-1 WSIAEs (1.1). Let $\left(y_{N}, z_{N}\right)$ be the spectral collocation approximation for the exact solution $(y, z)$ of system (1.1), and suppose the following conditions hold:

(1) $K_{1 j} \in C^{m}(D)(j=1,2), f_{1} \in C^{m}(I)$;

(2) $K_{2 j} \in C^{m+1}(D)(j=1,2), f_{2} \in C^{m+1}(I)$ with $f_{2}(0)=0$;

(3) $\left|K_{22}(v, v)\right|>0, \forall v \in I$.

Then for sufficiently large $N$,

$$
\begin{aligned}
& \left\|y_{N}-y\right\| \leq \begin{cases}O\left(N^{\frac{1}{2}-m} \log N\right), & -1<\alpha, \beta \leq-\frac{1}{2}, \\
O\left(N^{1+\gamma-m} \log N\right), & \text { otherwise, }\end{cases} \\
& \left\|z_{N}-z\right\| \leq \begin{cases}O\left(N^{\frac{5}{2}-m} \log N\right), & -1<\alpha, \beta \leq-\frac{1}{2}, \\
O\left(N^{3+\gamma-m} \log N\right), & \text { otherwise, }\end{cases}
\end{aligned}
$$

where $\gamma=\max \{\alpha, \beta\}$.

\section{Numerical experiments}

In $[14,19,21]$, the authors have analyzed the spectral Jacobi collocation method for Volterra integral equations of the second kind with singular kernel. Here, we consider 
the approximate solution for coupled system of weakly singular integral algebraic equations which consist of both the first and the second kind Volterra integral equations. To our knowledge, there are no other results as regards the numerical analysis of WSIAEs except [13], which designed an effective numerical method based on the Chebyshev collocation scheme for system (1.1) and provided its convergence analysis. It is noted that the Jacobi collocation method becomes the Chebyshev collocation method for $\alpha=\beta=-1 / 2$. In this section, some numerical examples are given to show the validity of the proposed method. Variable transformations (2.3) and linear transformations (3.1) are used to change the WSIAEs system into a new system such that its solutions have better regularity. All the computations were performed using the software Matla $b^{\circ}$. These problems are solved using the Jacobi collocation method for $\alpha=\frac{1}{3}, \beta=\frac{1}{2}$. In order to show the behavior of the errors, we define the weighted $L^{2}$-norm by

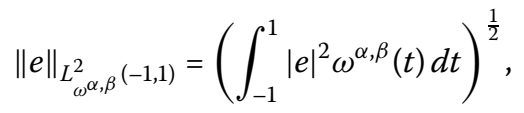

where $\omega^{\alpha, \beta}(x)=(1-x)^{\alpha}(1+x)^{\beta}(\alpha, \beta>-1)$ is the Jacobi weight function.

Example 1 Consider the following index-1 WSIAEs system:

$$
A X(t)=G(t)+\int_{0}^{t}(t-s)^{-\frac{1}{2}} K(t, s) X(s) d s, \quad t \in[0,1]
$$

where

$$
\begin{aligned}
& A=\left(\begin{array}{ll}
1 & 0 \\
0 & 0
\end{array}\right), \quad K(t, s)=\left(\begin{array}{cc}
t^{2}+1 & t+s^{2} \\
s^{2}+4 & t^{2}+s^{4}+1
\end{array}\right), \\
& X(t)=(y(t), z(t))^{T}, \quad F(t)=\left(f_{1}(t), f_{2}(t)\right)^{T},
\end{aligned}
$$

and $f_{1}, f_{2}$ are chosen such that the exact solution is

$$
y(t)=\arctan \sqrt{t}, \quad z(t)=\frac{e^{t}-1}{\sqrt{t}} .
$$

Because $y^{\prime}(t)=\frac{t^{-\frac{1}{2}}}{2(1+t)}, z^{\prime}(t)=t^{-\frac{1}{2}}\left(\frac{e^{t}-1}{2 t}+e^{t}\right)$, it is noted that $y^{\prime} \sim t^{-\frac{1}{2}}$ and $z^{\prime} \sim t^{-\frac{1}{2}}$ at $t \rightarrow 0$. For $\alpha=\frac{p}{q}=\frac{1}{2}$, by using variable transformation $t=u^{2}$, the smooth solutions $\hat{y}=y\left(u^{2}\right)$, $\hat{z}=z\left(u^{2}\right)$ are obtained. Inserting $\hat{y}, \hat{z}$ in the WSIAEs and using the transformation (3.1), we have

$$
A \tilde{X}(v)=\tilde{F}(v)+\int_{0}^{t}(v-x)^{-\frac{1}{2}} \tilde{K}(v, x) \tilde{X}(x) d x, \quad v \in[-1,1]
$$

where

$$
\tilde{K}(v, x)=\left(\begin{array}{ll}
\tilde{K}_{11}(v, x) & \tilde{K}_{12}(v, x) \\
\tilde{K}_{21}(v, x) & \tilde{K}_{22}(v, x)
\end{array}\right), \quad \tilde{X}(v)=(\tilde{y}(v), \tilde{z}(v))^{T}, \quad \tilde{F}(v)=\left(\tilde{f}_{1}(v), \tilde{f}_{2}(v)\right)^{T} .
$$


Table $1 L_{\omega^{\alpha, \beta}}^{2}(-1,1)$ errors for Example 1

\begin{tabular}{|c|c|c|}
\hline$N$ & $\left\|\tilde{y}_{N}-\tilde{y}\right\|_{L^{\alpha}{ }^{\alpha}, \beta}(-1,1)$ & $\left\|\tilde{z}_{N}-\tilde{z}\right\|_{L^{2}{ }^{\alpha, \beta}}(-1,1)$ \\
\hline 4 & $1.75 \times 10^{-4}$ & $1.61 \times 10^{-} 3$ \\
\hline 5 & $3.34 \times 10^{-5}$ & $2.59 \times 10^{-4}$ \\
\hline 6 & $7.33 \times 10^{-6}$ & $5.80 \times 10^{-5}$ \\
\hline 7 & $4.12 \times 10^{-7}$ & $5.62 \times 10^{-6}$ \\
\hline 8 & $2.35 \times 10^{-7}$ & $6.15 \times 10^{-6}$ \\
\hline 9 & $4.65 \times 10^{-8}$ & $3.96 \times 10^{-7}$ \\
\hline
\end{tabular}

Table $2 L_{\omega^{\alpha, \beta}}^{2}(-1,1)$ errors for Example 2

\begin{tabular}{|c|c|c|}
\hline$N$ & $\left\|\tilde{y}_{N}-\tilde{y}\right\|_{L^{2}{ }^{\alpha, \beta}}(-1,1)$ & $\left\|\tilde{z}_{N}-\tilde{z}\right\|_{L^{2}{ }^{\alpha, \beta}}(-1,1)$ \\
\hline 4 & $2.95 \times 10^{-4}$ & $2.84 \times 10^{-3}$ \\
\hline 5 & $4.55 \times 10^{-5}$ & $3.70 \times 10^{-4}$ \\
\hline 6 & $7.81 \times 10^{-6}$ & $8.62 \times 10^{-5}$ \\
\hline 7 & $4.72 \times 10^{-7}$ & $4.92 \times 10^{-6}$ \\
\hline 8 & $4.31 \times 10^{-7}$ & $8.78 \times 10^{-7}$ \\
\hline 9 & $5.29 \times 10^{-8}$ & $5.12 \times 10^{-7}$ \\
\hline
\end{tabular}

Let $\left(\tilde{y}_{N}, \tilde{z}_{N}\right)$ denote the approximation of the exact solution $(\tilde{y}, \tilde{z})$ that is given by equation (3.3). The proposed Jacobi collocation methods are applied for system (5.1). Table 1 shows the errors for $(\tilde{y}, \tilde{z})$. It is seen that the desired exponential rate of convergence is obtained.

\section{Example 2}

$$
A X(t)=G(t)+\int_{0}^{t}(t-s)^{-\frac{1}{4}} K(t, s) X(s) d s, \quad t \in[0,1],
$$

where

$$
\begin{aligned}
& A=\left(\begin{array}{ll}
1 & 0 \\
0 & 0
\end{array}\right), \quad K(t, s)=\left(\begin{array}{cc}
e^{\sqrt{s}+t}\left(t^{2}+s^{4}+3\right) & \cos s^{\frac{1}{4}}(t+s) \\
e^{\sqrt{s}+t^{2}+1}(t+s) & \sin \left(s^{\frac{1}{4}}+1\right)(t s+1)
\end{array}\right), \\
& X(t)=(y(t), z(t))^{T}, \quad G(t)=\left(f_{1}(t), f_{2}(t)\right)^{T},
\end{aligned}
$$

and $f_{1}, f_{2}$ are chosen such that the exact solution is

$$
y(t)=\exp (\sqrt{t}), \quad z(t)=\sin (\sqrt[4]{t}) .
$$

In Table 2 we present the weighted $L^{2}$-norm of errors for the numerical solutions by using the spectral collocation method.

\section{Conclusions}

This work has been concerned with the theoretical and numerical analysis of integral algebraic equations of index 1 with weakly singular kernels. It is noted that the solutions of the WSIAEs are not sufficiently smooth. So, the original system was changed into a new system, by using some function transformations and variable transformations. We presented a spectral Jacobi collocation approximation for the new WSIAEs. The error estimation of 
the method in the weighted $L^{2}$-norm was obtained. Numerical results are presented to confirm the theoretical prediction of the exponential rate of convergence.

\section{Competing interests}

The authors declare that they have no competing interests.

\section{Authors' contributions}

All authors contributed equally to the writing of this paper. All authors read and approved the final manuscript.

\section{Acknowledgements}

The authors would like to acknowledge the anonymous referees for their careful reading of the manuscript and constructive comments. This work is supported by the National Natural Science Foundation of China (11101109, 11271102), Natural Science Foundation of Hei-long-jiang Province of China (A201107) and SRF for ROCS, SEM.

Received: 21 January 2014 Accepted: 28 May 2014 Published: 05 Jun 2014

\section{References}

1. Cannon, JR: The One-Dimensional Heat Equation. Cambridge University Press, Cambridge (1984)

2. Gomilko, AM: A Dirichlet problem for the biharmonic equation in a semi-infinite strip. J. Eng. Math. 46, 253-268 (2003)

3. Goldman, NL: Inverse Stefan Problems. Mathematics and Its Applications, vol. 412. Kluwer Academic, Dordrecht (1997)

4. Slodička, M, Schepper, HD: Determination of the heat-transfer coefficient during solidification of alloys. Comput. Methods Appl. Mech. Eng. 194, 491-498 (2005)

5. Brunner, $\mathrm{H}$ : Collocation Methods for Volterra Integral and Related Functional Equations. Cambridge University Press, Cambridge (2004)

6. Bulatov, MV, Chistyakov, VF: The Properties of Differential-Algebraic Systems and Their Integral Analogs. Memorial University of Newfoundland, Newfoundland (1997)

7. Bulatov, MV: Regularization of singular system of Volterra integral equation. Comput. Math. Math. Phys. 42, 315-320 (2002)

8. Bulatov, MV, Lima, PM: Two-dimensional integral-algebraic systems: analysis and computational methods. J. Comput. Appl. Math. 236, 132-140 (2011)

9. Hadizadeh, M, Ghoreishi, F, Pishbin, S: Jacobi spectral solution for integral-algebraic equations of index-2. Appl. Numer. Math. 61, 131-148 (2011)

10. Pishbin, S, Ghoreishi, F, Hadizadeh, M: A posteriori error estimation for the Legendre collocation method applied to integral-algebraic Volterra equations. Electron. Trans. Numer. Anal. 38, 327-346 (2011)

11. Brunner, H, Bulatov, MV: On singular systems of integral equations with weakly singular kernels. In: Proceeding 11-th Baikal International School Seminar, pp. 64-67 (1998)

12. Bulatov, MV, Lima, PM, Weinmuller, E: Existence and uniqueness of solutions to weakly singular integral-algebraic and integro-differential equations. Vienna Technical University, ASC Report No. 21 (2012)

13. Pishbin, S, Ghoreishi, F, Hadizadeh, M: The semi-explicit Volterra integral algebraic equations with weakly singular kernels: the numerical treatments. J. Comput. Appl. Math. 245, 121-132 (2013)

14. Li, X, Tang, T: Convergence analysis of Jacobi spectral collocation methods for Abel-Volterra integral equations of second kind. Front. Math. China 7, 69-84 (2012)

15. Gear, CW: Differential algebraic equations, indices, and integral algebraic equations. SIAM J. Numer. Anal. 27 1527-1534 (1990)

16. Atkinson, K, Han, W: Theoretical Numerical Analysis: A Functional Analysis Framework. Springer, New York (2009)

17. Canuto, C, Hussaini, MY, Quarteroni, A, Zang, TA: Spectral Methods Fundamentals in Single Domains. Springer, Berlin (2006)

18. Graham, IG, Sloan, IH: Fully discrete spectral boundary integral methods for Helmholtz problems on smooth closed surfaces in $\mathrm{R}^{3}$. Numer. Math. 92, 289-323 (2002)

19. Chen, Y, Tang, T: Convergence analysis of the Jacobi spectral collocation methods for Volterra integral equations with a weakly singular kernel. Math. Comput. 79, 147-167 (2010)

20. Diogo, T, McKee, S, Tang, T: Collocation methods for second-kind Volterra integral equations with weakly singular kernels. Proc. R. Soc. Edinb. A 124, 199-210 (1994)

21. Chen, Y, Tang, T: Spectral methods for weakly singular Volterra integral equations with smooth solutions. J. Comput Appl. Math. 233, 938-950 (2009) 